\section{Congenital bilateral absence of the vas deferens and recombination at CFTR}

\author{
European Journal of Human Genetics (2006) 14, 801.
} doi:10.1038/sj.ejhg.5201622; published online 26 April 2006

Pompei et $a l^{1}$ report a paucity of variation on V470 haplotypes at the CFTR locus relative to M470 haplotypes on unselected chromosomes from the general population. A conservative estimate of the region of 'extended haplotype homozygosity' that they report would extend from I148T (29 kb $5^{\prime}$ of V470) to S1235R (68 kb 3' of V470). The authors suggest that this pattern could be explained by a recent increase of the V470 variant to high frequency, either by natural selection for V470 itself or for another, linked variant in cis. In this scenario, V470 haplotypes are depauperate for sequence variation because they have had little time to accumulate variation due to mutation or by recombination with $\mathrm{M} 470$ haplotypes.

Variation at the polymorphic repeats in intron 8 is of particular interest. Pompei et al found ( $\mathrm{tg})_{12}$ occurred on 16/76 (0.211) M470 haplotypes but only 8/218 (0.037) V470 haplotypes. Similarly, $t_{5}$ occurred on 4/76 (0.053) M470 haplotypes but only 2/218 (0.009) V470 haplotypes. This pattern of variation contrasts markedly with the haplotypes reported from men with congenital bilateral absence of the vas deferens (CBAVD). In a recent paper, ${ }^{2} 11$ of 13 men with CBAVD possessed a $(\mathrm{tg})_{12} \mathrm{t}_{5} \mathrm{~V} 470$ haplotype, and two possessed a $(\mathrm{tg})_{11} \mathrm{t}_{5} \mathrm{~V} 470$ haplotype, confirming the observation of Cuppens et $\mathrm{al}^{3}$ that $(\mathrm{tg})_{12} \mathrm{t}_{5} \mathrm{~V} 470$ is the most common $t_{5}$-bearing haplotype in men with CBAVD.

These data raise the possibility that men with CBAVD have a high frequency of chromosomes that have undergone recent recombination between $t_{5}$ in intron 8 and V470 in exon 9, and suggest an interaction in the disease process between variants on either side of the putative crossovers. The simplest interpretation would be that these variants are $(\operatorname{tg})_{12} t_{5}$ and V470 themselves. The former would result in low proportion of transcripts containing exon 9 and the latter in a translated gene product with reduced chloride channel activity. ${ }^{3}$ The selective elimination of recombinant haplotypes would contribute to maintaining the different patterns of genetic variation seen on M470 and V470 haplotypes.

David Haig

Department of Organismic and Evolutionary Biology, Harvard University, 26 Oxford Street, Cambridge, MA 02138, USA E-mail:dhaig@oeb.harvard.edu

\begin{abstract}
References
1 Pompei F, Ciminelli BM, Bombieri C et al: Haplotype block structure of the CFTR gene. Most variants are associated with the M470 allele in several European populations. Eur J Hum Genet 2006; 14: 85-93.

2 Stuppia L, Antonucci I, Binni F et al: Screening of mutations in the CFTR gene in 1195 couples entering assisted reproduction technique programs. Eur J Hum Genet 2005; 13: 959-964.

3 Cuppens H, Lin W, Jaspers $M$ et al: Polyvariant mutant cystic fibrosis transmembrane conductance regulator genes. The polymorphic (TG)m locus explains the partial penetrance of the T5 polymorphism as a disease mutation. I Clin Invest 1998; 101: 487-496.
\end{abstract}

\section{Reply to Professor Haig}

European Journal of Human Genetics (2006) 14, 801. doi:10.1038/sj.ejhg.5201621; published online 26 April 2006

D Haig offers the interesting suggestion that selective elimination of a recombinant CFTR gene TG12-T5-V470 haplotype would contribute to maintaining the different patterns of genetic variation seen on the M470 and the V470 haplotypes. He correctly indicates an increased frequency of the haplotype in CBAVD, and one might add in CF-like lung disease ${ }^{1}$ and nonclassic $\mathrm{CF}^{2}$ as well.

The effect of the selective elimination would be rather low though, as the haplotype would be eliminated only if: (1) it occurs in a male, and (2) it is compounded with a CF mutation (0.02). Therefore, the efficiency of transmission of this haplotype would be less than that of the other haplotypes, but just by $1 \%$.

He suggests that a recombination process might have occurred between T5 and V470 on the CFTR gene: we would like to indicate that also a replication slippage mechanism might be involved in a change of nucleotide repeat number to generate the haplotype.

Pier Franco Pignatti ${ }^{*}, 1$

${ }^{1}$ Section of Biology and Genetics, Department of Mother and Child and Biology-Genetics, University of Verona, Italy

${ }^{*}$ Correspondence: Dr PF Pignatti, Institute of Biology and Genetics, Department of Mother and Child and BiologyGenetics, University of Verona, Policlinico GB Rossi, Strada Le Grazie 8, Verona 37134, Italy. Tel: + 39045807 4403; Fax: + 39045580 111; E-mail: pierfranco.pignatti@univr.it

\section{References}

1 Noone PG, Pue CA, Zhou Z et al: Lung disease associated with the IVS8 5T allele of the CFTR gene. Am J Resp Crit Care Med 2000; 162: 1919_ 1924.

2 Groman JD, Hefferon TW, Casals T et al: Variation in a repeat sequence determines whether a common variant of the cystic fibrosis transmembrane conductance regulator gene is pathogenic or benign. Am J Hum Genet 2004; 74: 176-179. 